\title{
Edge Detection on DICOM Image using Triangular Norms in Type-2 Fuzzy
}

\author{
D. Nagarajan ${ }^{1}$, \\ M.Lathamaheswari ${ }^{2}$ \\ Department of Mathematics \\ Hindustan Institute of Technology \& \\ Science, Chennai-603 103, India
}

\author{
R.Sujatha ${ }^{3}$ \\ Department of Mathematics \\ S.S.N College of Engineering \\ Chennai-603 110, India.
}

\author{
J.Kavikumar ${ }^{4}$ \\ Department of Mathematics and \\ Statistics \\ Universiti Tun Hussein Onn, \\ Malaysia
}

\begin{abstract}
In image processing, edge detection is an important venture. Fuzzy logic plays a vital role in image processing to deal with lacking in quality of an image or imprecise in nature. This present study contributes an authentic method of fuzzy edge detection through image segmentation. Gradient of the image is done by triangular norms to extract the information. Triangular norms ( $T$ norms) and triangular conorms ( $T$ conorms) are specialized in dealing uncertainty. Therefore triangular norms are chosen with minimum and maximum operators for the purpose of morphological operations. Also, mathematical properties of aggregation operator to represent the role of morphological operations using Triangular Interval Type-2 Fuzzy Yager Weighted Geometric (TIT2FYWG) and Triangular Interval Type-2 Fuzzy Yager Weighted Arithmetic (TIT2FYWA) operators are derived. These properties represent the components of image processing. Here Edge detection is done for DICOM image by converting into $2 \mathrm{D}$ gray scale image, using Type-2 fuzzy MATLAB and which is the novelty of this work.
\end{abstract}

Keywords-Aggregation operators; $T$ norm; $T$ conorm; triangular interval type-2 fuzzy number (TIT2FN); fuzzy morphology; gray scale Image; medical image processing

\section{INTRODUCTION}

In the field of optimization problems in Mathematics, Statistics, Economics and Information Science, the max and min operators are very useful for any dimension. Uncertainty convoluted in most of the real world problems. Fuzzy theory has been developed as an efficient and powerful mechanism in mathematical design of many engineering and objective phenomena [1-5].To deal uncertainty in any field one needs an effective and predictable incentive. Usually incomplete data and errors in the analyzing stage will be the reason for getting vague situation and this can be dealt with fuzzy theory. Mathematical devices may figure out an impreciseness. The largest and the smallest elements of a precise set of real numbers is the maximum and the minimum and so Yager triangular norm is chosen for this work [6-10]. We are facing many problems to add, melt and synthesize the datum from different sources to get a conclusion. The operators may be chosen according to the characteristic properties and then the operations for minimum and maximum can be applied [11-14].

The triangular norms with maximum and minimum operators could be used for an image processing since these norms play as the synthesize operators for which these maximum and minimum operators are just an exclusive choice
[15-17]. A Fuzzy Set (FS) is defined from a universal set to [0, 1] and the membership values (MVs) of every element is a crisp value between 0 and 1 . This kind of system is called Type-1 Fuzzy Set (T1FS) system. In many of the real world problems it is necessary to have a MV itself fuzzy instead of crisp value which is called T2FS [18-19]. The generalization of union and intersection operators are triangular norms. Though the general case is important there is an equal important for the particular cases which provide efficient algorithm and more understanding missing in the general case [20].

T2FS is used when T1FS is blurred. In T2FS, the MVs lies in an interval so it is useful in image processing as many of the images are not properly visible. The parameter $\eta$ in Yager triangular norms, accepts for tuning the norm between the other norms [21]. Yager norms covers all the continuous norms by changing the parameter where as other major norms can't do the same and have more time complexity [22]. In automation, visual sense, remotely second scene analysis and bio medical image processing, Fuzzy image analysis has been applied. When the images with low brightness, the structure will not be evidently visible. In this situation, the sets which have better and naturally include different types of uncertainties might be useful for image analysis in any field.

To deal this complication Fuzzy Sets and their advanced extensions like T2FS Sets are suitable since it handles the uncertainty in a better way. Using Type-2 Fuzzy thresholding techniques, different regions and abnormal lesions can be separated. Image processing can be done by FMM using triangular norms. Using T2FS, collection of undesirable scraps can be made while noise exist. In image processing, image enrichment, clustering, thresholding, edge detection and morphological image processing are easy to be done using T2FS. Application of single image analysis is always not reliable and therefore image processing based on T2 Fuzzy system has been considered $[23,24]$. Borderline between two compatible regions is called an edge.

Using unit of the regional array, sense of the trial edge will be done at different points. Real world issues are levelheaded of various structures at various scales and an ideal image cannot be expected. The technique of selecting and detecting acute disruption in an image is called edge detection. DICOM is worn to store, transfer and pass on the medical images (MIs). Most of the MIs are saved in DICOM pattern where one can 
store data of an image and header as well and per file there is one slice in general. Singe color images are called gray scale which accommodate the knowledge of only gray level but not about color. Every pixel has some number of bits that determines available number of various gray levels [25-30].

The paper is organized in the following manner. In section II, literature review has been done related to the present work. In section III, basic definitions required for developing the concept have been described. In section IV, operational laws have been proposed for TIT2FN. In section V, aggregation properties have been proved using weighted arithmetic and geometric operators. In section VI, the theory of image processing and the role of T2FS and Yager norms is presented. In section VII, applied Type-2 fuzzy logic in edge detection for DICOM image in two dimensional through MATLAB. In section VIII, conclusion and future work is given.

\section{REVIEW OF LITERATURE}

The authors of, [1] described Aggregation operators elaborately with their advanced direction and applications. [2] explained about gathering of the information and its related aggregation operators. [3] proposed Frank Aggregation Operators (AOs) and its mathematical properties for TIT2FSs and applied in a decision making problem. [4] studied t norms of Yager and Hamacher and also metric space on fuzzy logic. [5] utilized AOs in the process of decision making under the environment of probabilistic fuzzy.

[17] proposed fuzzy image processing (FIP) using Dubois and Prade triangular norm. [22] proposed a methodology for an image condensation and rehabilitation on a Lossy image using fuzzy relational equations. [23] proposed a technique for image analysis with the application of morphological operators with the support of uninorms.

[24] described and explained very clearly about the role of theoretical fuzzy logic strategies in medical image processing. [25] reviewed the applications of type-2 fuzzy systems in the field of image processing. [26] presented a comprehensive depiction of imitation of an image with the help of fuzzy logic. [27] established an algorithm for edge detection under fuzzy environment where instability of a digital image for every pixel has been calculated.

[28] proposed a methodology for fusion of image under intuitionistic fuzzy setting. [29] introduced a new technique for edge detection with the support of representation of fuzzy image and pixels. [30] examined and done a comparative analysis of various techniques of edge detection. From this review it is found that there is no work has been done for edge detection on DICOM image using Type-2 fuzzy logic. This is the motivation of the present work.

\section{BASIC DEFINITIONS}

The following basic concepts are given for the better understanding of the paper.

\section{A. Aggregation Operator [3]}

Let $\left(M_{\alpha}\right)_{\alpha \in[0,1]}$ be a group of aggregation operators (AOs) which is non-decreasing. If $\mathrm{A}$ is an $\mathrm{AO}$ then

$$
M_{A}: \bigcup_{n \in N}[0,1]^{n} \rightarrow[0,1] .
$$

\section{B. Triangular Interval Type-2 Fuzzy Set (TIT2FS) [3]}

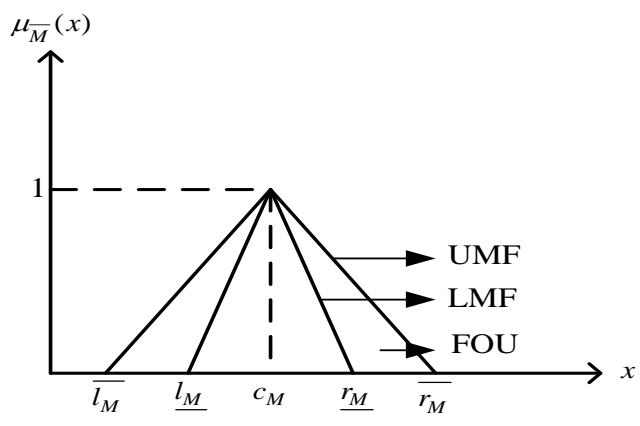

Fig. 1. TIT2FS.

The membership function (MFs) are developed using triangular fuzzy number in IT2FS called TIT2FS. In IT2FS, upper and lower MFs represented by a triangular fuzzy number $\bar{M}=\left\langle\left[\underline{l_{M}}, \overline{l_{M}}\right], c_{M},\left[\underline{r_{M}}, \overline{r_{M}}\right]\right\rangle$ called TIT2FS and are defined by

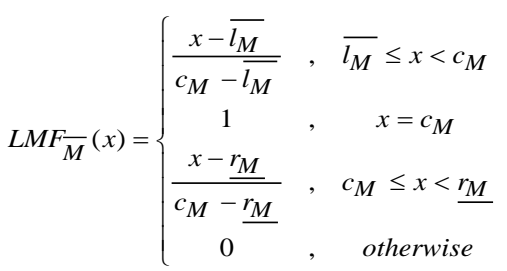

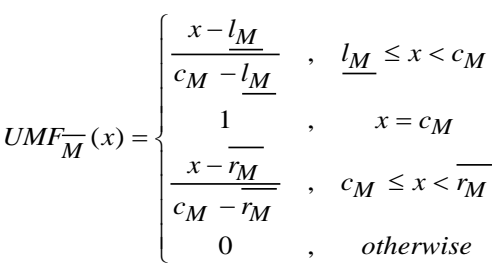

Where $l_{M}, \overline{l_{M}}, c_{M}, r_{M}, \overline{r_{M}}$ are the measuring points on TIT2FS satisfying $0 \leq \underline{l_{M}} \leq \overline{l_{M}} \leq c_{M} \leq \underline{r_{M}} \leq \overline{r_{M}} \leq 1$. If we consider $\boldsymbol{X}$ as a set of real numbers, a TIT2FS in $\boldsymbol{x}$ is called TIT2FN. The FOU is the area between lower and upper membership functions in figure 1. If $\underline{l_{M}}=\overline{l_{M}}, r_{M}=\overline{r_{M}}$, then $U M F_{\bar{M}}(x)=$ $L M F_{\bar{M}}(x)$ for all the values of $x$ in $x$, then the TIT2FS will become Type- 1 case. Here FOU is the footprint of Uncertainty.

\section{Ranking formula for TIT2FN [3]}

$$
\text { Let } \quad \bar{M}=\langle[A, B], C,[D, E]\rangle
$$

where

$A=\underline{l_{M}}, B=\overline{l_{M}}, C=c_{M}, D=\underline{r_{M}}, E=\overline{r_{M}}$ be the TIT2FN. The ranking value is defined by

$\operatorname{Rank}(\bar{M})=\left(\frac{A+E}{2}+1\right) \times \frac{A+B+D+E+4 C}{8}$ 


\section{Yager Triangular Norms [7]}

$\stackrel{\otimes}{Y}$ is Yager product (T Norm) and $\stackrel{\oplus}{Y}$ is a Yager sum

( $\mathrm{T}$ conorm) and are defined as follows.

$r \underset{Y}{\otimes} s=\max \left(1-\left[(1-r)^{\eta}+(1-s)^{\eta}\right]^{\frac{1}{\eta}}, 0\right), \eta>0$, for all $r, s \in[0,1]^{2}$

$\underset{Y}{\oplus} s=\min \left(\left(r^{\eta}+s^{\eta}\right)^{\frac{1}{\eta}}, 1\right), \eta>0$, for all $r, s \in[0,1]^{2}$

E. Triangular Interval Type-2 Fuzzy Yager Weighted Arithmetic (TIT2FYWA) Operator [3]

Consider a set of TIT2FNs and the operator $T I T 2 F Y W A_{\varepsilon}: \Omega^{n} \rightarrow \Omega$ is defined by TIT $2 F Y W A_{\varepsilon}\left\langle\overline{M_{1}}, \overline{M_{2}}, \ldots, \overline{M_{n}}\right\rangle=\varepsilon_{1} \bullet \overline{M_{1}} \underset{Y}{\oplus} \varepsilon_{2} \bullet \overline{M_{2}} \underset{Y}{\oplus} \ldots \underset{Y}{\oplus} \varepsilon_{n} \bullet \overline{M_{n}}$ and its weight vector is $\varepsilon=\left(\varepsilon_{1}, \varepsilon_{2}, \ldots, \varepsilon_{n}\right)^{T}$ and the sum of the weight vectors is equal to 1 , when $\varepsilon=(1 / n, 1 / n, \ldots, 1 / n)^{T}$, triangular interval type-2 fuzzy Yager weighted arithmetic operator will become triangular interval type-2 fuzzy Yager arithmetic averaging operator of dimension $n$ and is defined by

$$
\operatorname{TIT}_{2 F Y A A_{\varepsilon}}\left(\overline{M_{1}}, \overline{M_{2}}, \ldots, \overline{M_{n}}\right)=\frac{1}{n} \cdot\left(\overline{M_{1}} \underset{Y}{\oplus} \overline{M_{2}}, \ldots, \underset{Y}{\oplus} \overline{M_{n}}\right)
$$

F. Triangular Interval Type-2 Fuzzy Yager Weighted Geometric (TIT2FYWG) Operator [3]

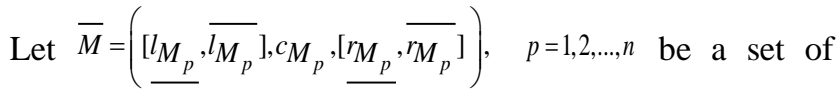
TIT2FNs. Triangular Interval Type-2 fuzzy Yager Weighted Geometric Mean Operator (TIT2FYWA), TIT2FYWG: $\varepsilon^{\mathrm{n}} \rightarrow \varepsilon$ is

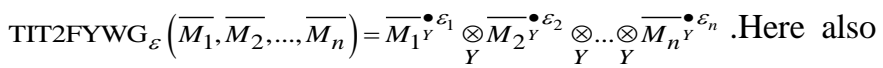
sum of all weight vectors is equal to 1 , when $\varepsilon=(1 / n, 1 / n, \ldots, 1 / n)^{T}$, triangular interval type-2 fuzzy Yager weighted arithmetic operator will become triangular interval type-2 fuzzy Yager geometric averaging operator of dimension $n$ and is defined by

$$
\left.\operatorname{TIT} 2 F Y G A_{\varepsilon}\left(\overline{M_{1}}, \overline{M_{2}}, \ldots, \overline{M_{2}}\right)=\frac{1}{n}\left(\overline{M_{1}} \underset{Y}{\otimes} \overline{M_{2}} \underset{Y}{\otimes}, \ldots, \underset{Y}{\otimes} \overline{M_{n}}\right) \dot{r}\right)^{1 / n}
$$

\section{PROPOSED Operational LAWS}

Let $\bar{M}, \bar{M}_{1}, \bar{M}_{2}$ be three TIT2FNs and $\eta>0$, then we define their operational laws as follows.

A. Addition

Consider, ${ }_{1}=\operatorname{sum}_{p=1}^{2}(\underbrace{l_{M}}_{M_{p}}), B_{1}=\underset{p=1}{2}\left(\overline{l_{M_{p}}}\right), C_{1}=\underset{p=1}{2} \operatorname{sum}_{p=1}\left(c_{M_{p}}\right)$,

$$
\begin{aligned}
& D_{1}=\underset{p=1}{2}\left(\underline{r_{M_{p}}}\right), E_{1}=\underset{p=1}{2}\left(\overline{r_{M_{p}}}\right) \\
& \overline{M_{1}} \underset{Y}{\oplus} \overline{M_{2}}=\left(\left[\min \left(A_{1}^{\frac{1}{\eta}}, 1\right), \min \left(B_{1}^{\frac{1}{\eta}}, 1\right)\right], \min \left(C_{1}^{\frac{1}{\eta}, 1}\right),\right. \\
& \left.\left[\min \left(D_{1}^{\frac{1}{\eta}}, 1\right), \min \left(E_{1}^{\frac{1}{\eta}}, 1\right)\right]\right)
\end{aligned}
$$

\section{B. Multiplication}

Consider,

$$
\begin{aligned}
& A_{2}=\underset{p=1}{\operatorname{sum}}\left(1-\underline{l_{M_{p}}}\right)^{\eta}, B_{2}=\underset{p=1}{\operatorname{sum}}\left(1-\overline{l_{M_{p}}}\right)^{\eta}, \\
& C_{2}=\underset{p=1}{2}\left(1-c_{M_{p}}\right)^{\eta}, \\
& D_{2}=\underset{p=1}{2} \operatorname{sum}_{p=1}^{2}\left(1-\underline{r_{M_{p}}}\right)^{\eta}, E_{2}=\operatorname{sum}_{p=1}^{2}\left(1-\overline{r_{M_{p}}}\right)^{\eta} . \\
& \overline{M_{1}} \underset{Y}{\otimes M_{2}}=\left\{\left[\max \left(1-A_{2}{ }^{\frac{1}{\eta}}, 0\right), \max \left(1-B_{2}{ }^{\frac{1}{\eta}}, 0\right)\right],\right. \\
& \left.\max \left(1-C_{2}{ }^{\frac{1}{\eta}}, 0\right),\left[\max \left(1-D_{2}{ }^{\frac{1}{\eta}}, 0\right), \max \left(1-E_{2}^{\frac{1}{\eta}}, 0\right)\right]\right\}
\end{aligned}
$$

C. Multiplication by an ordinary number

$$
\text { Consider, } A=\underline{l_{M}}, B=\overline{l_{M}}, C=c_{M}, D=\underline{r_{M}}, E=\overline{r_{M}}
$$

$$
\begin{aligned}
& \underset{Y}{\underset{\oplus}{\oplus} \bar{M}}=\left\{\left[\min \left\langle A^{\frac{k}{\eta}}, 1\right\rangle, \min \left\langle B^{\frac{k}{\eta}}, 1\right\rangle\right]\right. \\
& \min \left\langle C^{\frac{k}{\eta}}, 1\right\rangle,\left[\min \left\langle D^{\frac{k}{\eta}}, 1\right\rangle, \min \left\langle E^{\frac{k}{\eta}}, 1\right\rangle\right\}
\end{aligned}
$$

\section{Power}

Consider

$$
\begin{aligned}
& A_{3}=1-\underline{l_{M}}, B_{3}=1-\overline{l_{M}}, C_{3}=1-c_{M}, D_{3}=1-\underline{r_{M}}, E_{3}=1-\overline{r_{M}} \\
& \bar{M}^{\hat{Y}} k=\left\{\left[\max \left(1-\left[A_{3}{ }^{\eta}\right]^{k / \eta}, 0\right), \max \left(1-\left[B_{3}{ }^{\eta}\right]^{k / \eta}, 0\right)\right],\right. \\
& \max \left(1-\left[C_{3}{ }^{\eta}\right]^{k / \eta}, 0\right),
\end{aligned}
$$


$\left.\left[\max \left(1-\left[D_{3}{ }^{\eta}\right]^{k / \eta}, 0\right), \max \left(1-\left[E_{3}{ }^{\eta}\right]^{k / \eta}, 0\right)\right]\right\}$

\section{PROPOSED THEOREMS}

Here the mathematical properties of aggregation properties for TIT2FN using TIT2FYWG and TIT2FYWA operators are proved and they are playing an important role in image processing.

Consider a collection of TIT2FNs $\bar{M}=\left(\left[\underline{l_{M_{p}}}, \overline{l_{M_{p}}}\right], c_{M_{p}},\left[\underline{r_{M_{p}}}, \overline{r_{M_{p}}}\right]\right), \quad p=1,2, \ldots, n$

Where $0 \leq \underline{l_{M}} \leq \overline{l_{M}} \leq c_{M} \leq \underline{r_{M}} \leq \overline{r_{M}} \leq 1$

\section{A. Theorem}

The aggregation value of these fuzzy numbers using TIT2FYWG operator is again a TIT2FN and

$\operatorname{TIT} 2 F Y W G_{\varepsilon}\left\langle\overline{M_{1}}, \overline{M_{2}}, \ldots, \overline{M_{n}}\right\rangle=\left\{\left[\max \left(1-\left[A_{n}{ }^{\eta}\right]^{\varepsilon_{p} / \eta}, 0\right)\right.\right.$,

$\left.\max \left(1-\left[B_{n}{ }^{\eta}\right]^{\varepsilon_{p} / \eta}, 0\right)\right], \max \left(1-\left[C_{n}{ }^{{ }^{2}}\right]^{\varepsilon_{p} / \eta}, 0\right)$,

$\left.\left[\max \left(1-\left[D_{n}{ }^{\eta}\right]^{\varepsilon_{p} / \eta}, 0\right), \max \left(1-\left[E_{n}{ }^{\eta}\right]^{\varepsilon_{p} / \eta}, 0\right)\right]\right\}$

Where the weight vector is $\varepsilon=\left(\varepsilon_{1}, \varepsilon_{2}, \ldots, \varepsilon_{n}\right)^{T}, \varepsilon_{n} \geq 0$,

the sum of the weight vectors is equal to 1 .

Proof:

Here use mathematical induction method.

Case (i): For $n=2$.

Consider,

$$
P_{1}=\left(1-\underline{l_{M_{1}}}\right)^{\eta}, Q_{1}=\left(1-\overline{l_{M_{1}}}\right)^{\eta}, R_{1}=\left(1-c_{M_{1}}\right)^{\eta}
$$

$S_{1}=\left(1-\underline{r_{M_{1}}}\right)^{\eta}, T_{1}=\left(1-\overline{r_{M_{1}}}\right)^{\eta}$

Using Yager power operation

${\overline{M_{1}}}^{\dot{Y}_{k}}=\left\{\left[\max \left(1-P_{1}^{\frac{k}{\eta}}, 0\right), \max \left(1-Q_{1}^{\frac{k}{\eta}}, 0\right)\right]\right.$,

$\left.\max \left(1-R_{1}^{\frac{k}{\eta}}, 0\right),\left[\max \left(1-S_{1}^{\frac{k}{\eta}}, 0\right), \max \left(1-T_{1}^{\frac{k}{\eta}}, 0\right)\right]\right\}$
Consider,

$$
P_{2}=\left(1-\underline{l_{M_{1}}}\right)^{\eta}, Q_{2}=\left(1-\overline{l_{M_{1}}}\right)^{\eta}, R_{2}=\left(1-c_{M_{1}}\right)^{\eta}
$$

$S_{2}=\left(1-\underline{r_{M_{1}}}\right)^{\eta}, T_{2}=\left(1-\overline{r_{M_{1}}}\right)^{\eta}$
${\overline{M_{2}}}^{\dot{Y}} k=\left\{\left[\max \left(1-P_{2}^{\frac{k}{\eta}}, 0\right), \max \left(1-Q_{2}^{\frac{k}{\eta}}, 0\right)\right.\right.$,

$\left.\max \left(1-R_{2}{ }^{\frac{k}{\eta}}, 0\right),\left[\max \left(1-S_{2}^{\frac{k}{\eta}}, 0\right), \max \left(1-T_{2}^{\frac{k}{\eta}}, 0\right)\right]\right\}$.

$\operatorname{TIT} 2 F Y W G_{\mathcal{E}}\left(\overline{M_{1}}, \overline{M_{2}}\right)=\overline{M_{1}} \stackrel{\bullet}{\varepsilon_{1}} \underset{Y}{\otimes} \overline{M_{2}} \cdot{ }^{Y} \varepsilon_{2}$

$=\left\{\left[\max \left(1-\left[\underset{p=1}{2}\left(1-\max \left(1-\left(1-A_{2}\right) \frac{\varepsilon_{p}}{\eta}\right), 0\right)\right], 0\right)\right.\right.$,

$\max \left(1-\left[\underset{p=1}{2} \underset{p u m}{\sin }\left(1-\max \left(1-\left(1-B_{2}\right) \frac{\varepsilon_{p}}{\eta}\right), 0\right)\right], 0\right)$,

$\max \left(1-\left[\underset{p=1}{2} \underset{p=1}{\operatorname{sim}}\left(1-\max \left(1-\left(1-C_{2}\right) \frac{\varepsilon_{p}}{\eta}\right), 0\right)\right], 0\right)$,

$\left[\max \left(1-\left[\underset{p=1}{2}\left(1-\max \left(1-\left(1-D_{2}\right) \frac{\varepsilon_{p}}{\eta}\right), 0\right)\right], 0\right)\right.$,

$\left.\left.\max \left(1-\left[\underset{p=1}{2}\left(1-\max \left(1-\left(1-E_{2}\right) \frac{\varepsilon_{p}}{\eta}\right), 0\right)\right], 0\right)\right]\right\}$.

$A_{4}=\left(1-\underline{l_{M_{p}}}\right)^{\eta}, B_{4}=\left(1-\overline{l_{M_{p}}}\right)^{\eta}, C_{4}=\left(1-c_{M_{p}}\right)^{\eta}$,

$D_{4}=\left(1-\underline{r_{M_{p}}}\right)^{\eta}, E_{4}=\left(1-\overline{r_{M_{p}}}\right)^{\eta}$

$=\left\{\left[\max \left(1-\left(\underset{p=1}{2}\left(1-\max \left(1-A_{4}^{\frac{\varepsilon_{p}}{\eta}}, 0\right)\right), 0\right)\right.\right.\right.$,

$\max \left(1-\left(\underset{p=1}{2}\left(1-\max \left(1-B_{4}^{\frac{\varepsilon_{p}}{\eta}}, 0\right)\right), 0\right)\right)$, 


$$
\begin{aligned}
& \max \left(1-\left(\underset{p=1}{2}\left(1-\max \left(1-C_{4}^{\frac{\varepsilon_{p}}{\eta}}, 0\right)\right), 0\right),\right. \\
& {\left[\operatorname { m a x } \left(1-\left(\underset{p=1}{2}\left(1-\max \left(1-D_{4}^{\frac{\varepsilon_{p}}{\eta}}, 0\right)\right), 0\right),\right.\right.} \\
& \left.\max \left(1-\left(\underset{p=1}{2}\left(1-\max \left(1-E_{4}^{\frac{\varepsilon_{p}}{\eta}}, 0\right)\right), 0\right)\right)\right\} \\
& =\left\{\left[\max \left[1-A_{2}^{\frac{\varepsilon_{p}}{\eta}}, 0\right], \max \left[1-B_{2}^{\frac{\varepsilon_{p}}{\eta}}, 0\right], \max \left[1-C_{2}^{\frac{\varepsilon_{p}}{\eta}}, 0\right],\right.\right. \\
& {\left[\max \left[1-D_{2}^{\frac{\varepsilon_{p}}{\eta}}, 0\right], \max \left[1-E_{2}^{\frac{\varepsilon_{p}}{\eta}}, 0\right]\right\}}
\end{aligned}
$$

For $n=k$,

$$
\begin{aligned}
& A_{k}=\operatorname{sum}_{p=1}^{k}\left(1-\underline{l_{M_{p}}}\right)^{\eta}, B_{k}=\underset{p=1}{k}\left(1-\overline{l_{M_{p}}}\right)^{\eta}, C_{k}=\underset{p=1}{k}\left(1-c_{M_{p}}\right)^{\eta}, \\
& D_{k}=\underset{p=1}{k}\left(1-\underline{r_{M_{p}}}\right)^{\eta}, E_{k}=\underset{p=1}{k}\left(1-\overline{r_{M_{p}}}\right)^{\eta} \text {. } \\
& T I T 2 F Y W G_{\varepsilon}\left\langle\overline{M_{1}}, \overline{M_{2}}, \ldots, \overline{M_{k}}\right\rangle \\
& =\left\{\left[\operatorname { m a x } \left(1-\left[\underset{p=1}{k}\left(1-\max \left(1-\left[A_{k}\right]^{\frac{\omega_{p}}{\eta}}, 0\right)\right], 0\right),\right.\right.\right. \\
& \max \left(1-\left[\underset{p=1}{k}\left(1-\max \left(1-B_{k}^{\frac{\omega_{p}}{\eta}}, 0\right)\right], 0\right),\right. \\
& \max \left(1-\left[\underset{p=1}{k}\left(1-\max \left(1-C_{k}^{\frac{\omega_{p}}{\eta}}, 0\right)\right], 0\right),\right. \\
& {\left[\operatorname { m a x } \left(1-\left[\underset{p=1}{k}\left(1-\max \left(1-D_{k}^{\frac{\varepsilon_{p}}{\eta}}, 0\right)\right], 0\right),\right.\right.} \\
& \left.\max \left(1-\left[\underset{p=1}{k}\left(1-\max \left(1-E_{k}^{\left.\frac{\varepsilon_{p}}{\eta}, 0\right)}\right)\right], 0\right)\right]\right\} \text {. }
\end{aligned}
$$

$$
\begin{aligned}
=\left\{\left[\max \left[1-A_{k}^{\frac{\varepsilon_{p}}{\eta}}, 0\right], \max \left[1-B_{k}^{\frac{\varepsilon_{p}}{\eta}}, 0\right],\right.\right. \\
\max \left[1-C_{k}^{\frac{\varepsilon_{p}}{\eta}}, 0\right],\left[\max \left[1-D_{k}^{\frac{\varepsilon_{p}}{\eta}}, 0\right], \max \left[1-E_{k}^{\frac{\varepsilon_{p}}{\eta}}, 0\right]\right\} .
\end{aligned}
$$$$
\text { For } n=k+1 \text {, }
$$$$
\operatorname{TIT} 2 F Y W G_{\varepsilon}\left\langle\overline{M_{1}}, \overline{M_{2}}, \ldots, \overline{M_{k}}\right\rangle \underset{Y}{\otimes} T I T 2 F Y W G_{\varepsilon}\left\langle\overline{M_{k+1}}\right\rangle
$$$$
=\left\{\left[\operatorname { m a x } \left(1-\left[\underset{p=1}{k}\left(1-\max \left(1-A_{k}^{\frac{\varepsilon_{p}}{\eta}}, 0\right)\right], 0\right),\right.\right.\right.
$$$$
\max \left(1-\left[\underset{p=1}{k}\left(1-\max \left(1-B_{k}^{\frac{\varepsilon_{p}}{\eta}}, 0\right)\right], 0\right)\right],
$$$$
\max \left(1-\left[\underset{p=1}{k}\left(1-\max \left(1-C_{k}^{\frac{\varepsilon_{p}}{\eta}}, 0\right)\right], 0\right),\right.
$$$$
\left[\operatorname { m a x } \left(1-\left[\underset{p=1}{k}\left(1-\max \left(1-D_{k}^{\frac{\varepsilon_{p}}{\eta}}, 0\right)\right], 0\right)\right.\right. \text {, }
$$$$
\left.\max \left(1-\left[\underset{p=1}{k}\left(1-\max \left(1-E_{k}^{\frac{\varepsilon_{p}}{\eta}}, 0\right)\right], 0\right)\right]\right\} \text {. }
$$$$
\underset{Y}{\otimes}\left\{\left[\max \left(1-P_{k+1}^{\frac{\omega_{k+1}}{\eta}}, 0\right), \max \left(1-Q_{k+1}^{\frac{\varepsilon_{k+1}}{\eta}}, 0\right)\right],\right.
$$$$
\max \left(1-R_{k+1}^{\frac{\varepsilon_{k+1}}{\eta}}, 0\right),
$$$$
\left.\left[\max \left(1-S_{k+1}^{\frac{\varepsilon_{k+1}}{\eta}}, 0\right), \max \left(1-T_{k+1}^{\frac{\varepsilon_{k+1}}{\eta}}, 0\right)\right]\right\} .
$$$$
=\left\{\left[\max \left(1-\left[\underset{p=1}{k+1}\left(1-\max \left(1-A_{k}^{\frac{\varepsilon_{p}}{\eta}}\right), 0\right)\right], 0\right),\right.\right.
$$ 


$$
\begin{aligned}
& \left.\max \left(1-\left[\underset{p=1}{k+1}\left(1-\max \left(1-B_{k}^{\frac{\varepsilon_{p}}{\eta}}\right), 0\right)\right], 0\right)\right] \\
& \max \left(1-\left[\underset{p=1}{k+1}\left(1-\max \left(1-C_{k}^{\frac{\varepsilon_{p}}{\eta}}\right), 0\right), 0\right)\right. \text {, } \\
& {\left[\max \left(1-\left[\underset{p=1}{k+1}\left(1-\max \left(1-D_{k}^{\frac{\varepsilon_{p}}{\eta}}\right), 0\right)\right], 0\right),\right.} \\
& \left.\left.\max \left(1-\left[\underset{p=1}{k+1}\left(1-\max \left(1-E_{k}^{\frac{\varepsilon_{p}}{\eta}}\right), 0\right)\right], 0\right)\right]\right\} \text {. } \\
& =\left\{\left[\max \left[1-A_{k+1}^{\frac{\varepsilon_{p}}{\eta}}, 0\right], \max \left[1-B_{k+1}^{\frac{\varepsilon_{p}}{\eta}}, 0\right]\right.\right. \text {, } \\
& \max \left[1-C_{k+1}^{\frac{\varepsilon_{p}}{\eta}}, 0\right],\left[\max \left[1-D_{k+1}^{\frac{\omega_{p}}{\eta}}, 0\right], \max \left[1-E_{k+1}^{\frac{\varepsilon_{p}}{\eta}}, 0\right]\right\} \text {. }
\end{aligned}
$$

Hence the result holds for all the values of $n$.

\section{B. Theorem(Idempotency)}

If $\overline{M_{p}}=\bar{M}$ for all the values of $p$ then $\operatorname{TIT} 2 F Y W G_{\varepsilon}\left\langle\overline{M_{1}}, \overline{M_{2}}, \ldots, \overline{M_{n}}\right\rangle=\bar{M}$.

Proof:

By theorem A,

$$
\begin{aligned}
& \text { TIT } 2 F Y W G_{\varepsilon}\left\langle\overline{M_{1}}, \overline{M_{2}}, \ldots, \overline{M_{n}}\right\rangle \\
&=\left\{\left[\max \left(1-A_{n}^{\frac{\varepsilon_{p}}{\eta}}, 0\right), \max \left(1-B_{n}^{\frac{\omega_{p}}{\eta}}, 0\right)\right],\right. \\
&\left.\max \left(1-C_{n}^{\frac{\varepsilon_{p}}{\eta}}, 0\right),\left[\max \left(1-D_{n}^{\frac{\varepsilon_{p}}{\eta}}, 0\right), \max \left(1-E_{n}^{\frac{\varepsilon_{p}}{\eta}}, 0\right)\right]\right\} .
\end{aligned}
$$$$
\operatorname{TIT} 2 F Y W G_{\varepsilon}\left\langle\overline{M_{1}}, \overline{M_{2}}, \ldots, \overline{M_{n}}\right\rangle
$$

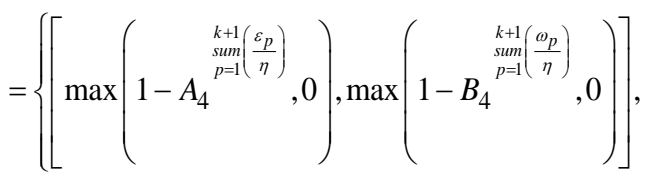

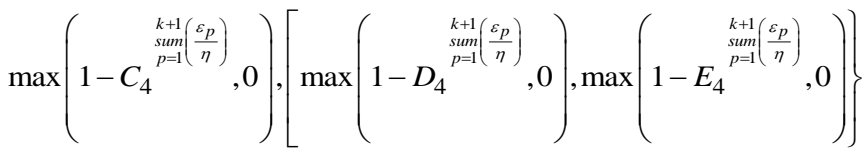

$$
\begin{aligned}
& =\left\{\left[\max \left(1-A_{5}^{\frac{1}{\eta}}, 0\right), \max \left(1-B_{5}^{\frac{1}{\eta}}, 0\right)\right]\right. \text {, } \\
& \left.\max \left(1-C_{5}^{\frac{1}{\eta}}, 0\right),\left[\max \left(1-D_{5}^{\frac{1}{\eta}}, 0\right), \max \left(1-E_{5}^{\frac{1}{\eta}}, 0\right)\right]\right\} . \\
& =\left\{\left[A_{3}, B_{3}, C_{3}, D_{3}, E_{3}\right]\right\}=\{[A, B], C,[D, E]\}=\bar{M} \\
& \text { C. Theorem(Boundary) } \\
& \text { Let } \\
& \bar{M}^{+}=\left\{\left[\underset{p=1}{n}\left(\underline{l_{M_{p}}}\right), \max _{p=1}^{n}\left(\overline{l_{M_{p}}}\right)\right], \underset{p=1}{n} \max _{M_{p}},\left[\underset{p=1}{n}\left(\underline{r_{M_{p}}}\right), \max _{p=1}^{n}\left(\overline{r_{M_{p}}}\right)\right]\right\}
\end{aligned}
$$

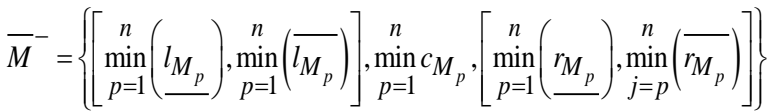

$$
\begin{aligned}
& \text { Then } \bar{M}^{-} \leq \text {TIT } 2 F Y W G_{\varepsilon}\left\langle\overline{M_{1}}, \overline{M_{2}}, \ldots, \bar{M}_{n}\right\rangle \leq \bar{M}^{+}
\end{aligned}
$$

Proof:

Since,

$$
\begin{aligned}
& \min _{p=1}^{n}\left(\underline{l_{M_{p}}}\right) \leq \underline{l_{M_{p}}} \leq \max _{p=1}^{n}\left(\underline{l_{M_{p}}}\right), \underset{p=1}{\min }\left(\overline{l_{M_{p}}}\right) \leq \overline{l_{M_{p}}} \leq \max _{p=1}^{n}\left(\overline{l_{M_{p}}}\right), \\
& \min _{p=1}^{n}\left(c_{M_{p}}\right) \leq c_{M_{p}} \leq \max _{p=1}^{n}\left(c_{M_{p}}\right), \\
& \min _{p=1}^{n}\left(\underline{r_{M_{p}}}\right) \leq{\underline{r_{M_{p}}}}^{n} \max _{p=1}^{n}\left(\underline{r_{M_{p}}}\right), \min _{p=1}^{n}\left(\overline{r_{M_{p}}}\right) \leq \overline{r_{M_{p}}} \leq \max _{j=1}^{n}\left(\overline{r_{M_{p}}}\right) .
\end{aligned}
$$

we have,

$$
\begin{aligned}
& 1-\max _{p=1}^{n}\left(\underline{l_{M_{p}}}\right) \leq \underline{l_{M_{p}}} \leq 1-\min _{p=1}^{n}\left(\underline{l_{M_{p}}}\right) \\
& \Rightarrow \min \left(\left[\underset{p=1}{n}\left(1-\max \left(\underline{l_{M_{p}}}\right)\right)^{\eta}\right]^{\frac{1}{\eta}}, 1\right) \leq \min \left(\left[\underset{p=1}{n}\left(1-\left(\underline{l_{M_{p}}}\right)\right)^{\eta}\right]^{\frac{1}{\eta}}, 1\right) \\
& \leq \min \left(\left[\underset{p=1}{\operatorname{sum}}\left(1-\min \left(\underline{l_{M_{p}}}\right)\right)^{\eta}\right]^{\frac{1}{\eta}}, 1\right) .
\end{aligned}
$$




$$
\begin{aligned}
& \Rightarrow \min \left(\left[\left(1-\max \left(\underline{l_{M p}}\right)\right)^{\eta}\right]_{\substack{n \\
p=1}}^{p r o d}\left(\frac{1}{\eta}\right)\right) \leq \min \left(\left[\left(1-\underline{l_{M_{p}}}\right)^{\gamma}\right]_{\substack{p r o d \\
p=1}}^{p\left(\frac{1}{\eta}\right)}, 1\right) \\
& \leq \min \left(\left[\left(1-\min \left(\underline{l_{M_{p}}}\right)\right)^{\eta}\right]_{\substack{p r o d \\
p=1}}^{\stackrel{n}{\eta}\left(\frac{1}{\eta}\right)}, 1\right) \\
& \Rightarrow \min \left(\left[\left(1-\max \left(\underline{l_{M_{p}}}\right)\right)\right], 1\right) \leq \min \left(\left[\left(1-\underline{l_{M_{p}}}\right)\right], 1\right) \\
& \leq \min \left(\left[\left(1-\min \left(\underline{l_{M_{p}}}\right)\right)\right], 1\right) \\
& \Rightarrow \min \left(\min \left(\underline{l_{M_{p}}}\right), 1\right) \leq \min \left(1-\underset{p=1}{n}\left(\underline{l_{M_{p}}}\right), 1\right) \leq \min \left(\max \left(\underline{l_{M_{p}}}\right), 1\right) \\
& \Rightarrow \min \left(\underline{l_{M_{p}}}\right) \leq \min \left(1-\underset{p=1}{n}\left(\underline{l_{M_{p}}}\right), 1\right) \leq \max \left(\underline{l_{M_{p}}}\right)
\end{aligned}
$$

Similarly we have,

$\min \left(\overline{l_{M_{p}}}\right) \leq \min \left(1-\operatorname{sum}_{p=1}^{n}\left(\overline{l_{M_{p}}}\right), 1\right) \leq \max \left(\overline{l_{M_{p}}}\right)$,

$\min \left(c_{M_{p}}\right) \leq \min \left(1-\operatorname{sum}_{p=1}^{n}\left(c_{M_{p}}\right), 1\right) \leq \max \left(c_{M_{p}}\right)$

$\min \left(\underline{r_{M_{p}}}\right) \leq \min \left(1-\underset{p=1}{n}\left(\underline{r_{M_{p}}}\right), 1\right) \leq \max \left(\underline{r_{M_{p}}}\right)$

$\min \left(\overline{r_{M_{p}}}\right) \leq \min \left(1-\operatorname{sum}_{p=1}^{n}\left(\overline{l_{M_{p}}}\right), 1\right) \leq \max \left(\overline{r_{M_{p}}}\right)$

By using the ranking value formula for TIT2FN and using the arithmetic average ranking value,

$$
\begin{aligned}
& R(\bar{M})=\left(\frac{\frac{l_{M_{p}}}{\underline{r_{M_{p}}}}}{2}+1\right) \times \frac{l_{M_{p}}+\overline{l_{M_{p}}}+\frac{r_{M_{p}}}{\frac{r_{M_{p}}}{4}+4 c_{M}}}{8} \\
& \leq\left(\frac{\max \left(\frac{l_{M_{p}}}{}\right)+\max \left(\overline{r_{M_{p}}}\right)}{2}+1\right) \times\left(\max _{p=1}^{n}\left(\underline{l_{M_{p}}}\right)+\max _{p=1}^{n}\left(\overline{l_{M_{p}}}\right)+\max _{p=1}^{n}\left(\underline{r_{M_{p}}}\right)\right) \\
& \left.+\max _{p=1}^{n}\left(\overline{r_{M_{p}}}\right)+4 \max _{p=1}^{n}\left(c_{M_{p}}\right)\right) \times 8^{-1}=R\left(\bar{M}^{+}\right)
\end{aligned}
$$

Hence the result.

\section{Theorem}

If $t>0$ for all the values of $\mathrm{p}$ then

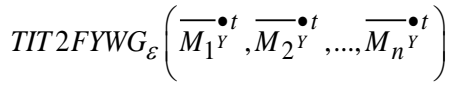

$=T I T 2 F Y W G_{\varepsilon}\left(\overline{M_{1}}, \overline{M_{2}}, \ldots, \overline{M_{n}}\right)^{\bullet t}$

Proof:

$$
\begin{aligned}
& \bar{M}^{\bullet} \cdot t=\left\{\left[\max \left(1-A_{5}^{\frac{t}{\eta}}, 0\right), \max \left(1-B_{5}^{\frac{t}{\eta}}, 0\right)\right], \max \left(1-C_{5}^{\frac{t}{\eta}}, 0\right),\right. \\
& \left.\left.\max \left(1-D_{5}^{\frac{t}{\eta}}, 0\right), \max \left(1-E_{5}^{\frac{t}{\eta}}, 0\right)\right]\right\} . \\
& =\left\{\left[\max \left(1-\left[\begin{array}{c}
k \\
\operatorname{sum} \\
p=1
\end{array}\left(1-\max \left(1-A_{k}^{\frac{\varepsilon_{p}}{\eta}}, 0\right)\right)\right], 0\right),\right.\right. \\
& \max \left(1-\left[\underset{k=1}{\operatorname{sum}}\left(1-\max \left(1-B_{k}^{\frac{\varepsilon_{p}}{\eta}}, 0\right)\right)\right], 0\right],
\end{aligned}
$$$$
\max \left(1-\left[\underset{p=1}{\operatorname{sum}}\left(1-\max \left(1-C_{k}^{\frac{\varepsilon_{p}}{\eta}}, 0\right)\right)\right], 0\right),
$$$$
\left[\operatorname { m a x } \left(1-\left[\underset{p=1}{k}\left(1-\max \left(1-D_{k}^{\frac{\varepsilon_{p}}{\eta}}, 0\right)\right], 0\right),\right.\right.
$$$$
\left.\left.\max \left(1-\left[\underset{p=1}{k}\left(1-\max \left(1-E_{k}^{\frac{\varepsilon_{p}}{\eta}, 0}\right)\right)\right], 0\right)\right]\right\} .
$$

$\operatorname{TIT} 2 F Y W G_{\varepsilon}\left\langle\overline{M_{1}}, \overline{M_{2}}, \ldots, \overline{M_{n}}\right\rangle$

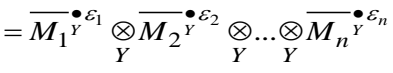

$=\left\{\left[\max \left(1-\left[\underset{p=1}{n}\left(1-\max \left(1-\left(1-A_{2}\right) \frac{\varepsilon_{p}}{\eta}\right), 0\right)\right], 0\right)\right.\right.$,

$\left.\max \left(1-\left[\underset{p=1}{n}\left(1-\max \left(1-\left(1-B_{2}\right) \frac{\varepsilon_{p}}{\eta}\right), 0\right)\right], 0\right)\right]$

$\max \left(1-\left[\underset{p=1}{n}\left(1-\max \left(1-\left(1-C_{2}\right) \frac{\varepsilon_{p}}{\eta}\right), 0\right)\right], 0\right)$ 


$$
\begin{aligned}
& {\left[\max \left(1-\left[\operatorname{sum}_{p=1}^{n}\left(1-\max \left(1-\left(1-D_{2}\right)^{\frac{\varepsilon_{p}}{\eta}}\right), 0\right)\right], 0\right),\right.} \\
& \left.\left.\quad \max \left(1-\left[\operatorname{sum}_{p=1}^{n}\left(1-\max \left(1-\left(1-E_{2}\right)^{\frac{\varepsilon_{p}}{\eta}}\right), 0\right)\right], 0\right)\right]\right\} . \\
& =\left\{\left[\max \left(1-A_{n}^{\frac{t \varepsilon_{p}}{\eta}, 0}, \max \left(1-B_{n}^{\frac{t \varepsilon_{p}}{\eta}}, 0\right)\right],\right.\right. \\
& \left.\max \left(1-C_{n}^{\frac{t \varepsilon_{p}}{\eta}}, 0\right),\left[\max \left(1-D_{n}^{\frac{t \varepsilon_{p}}{\eta}}, 0\right), \max \left(1-E_{n}^{\frac{t \varepsilon_{p}}{\eta}, 0}\right)\right]\right\} .
\end{aligned}
$$

Also since,

$$
\begin{aligned}
& \operatorname{TIT} 2 F Y W G_{\varepsilon}\left(\overline{M_{1}}, \overline{M_{2}}, \ldots, \overline{M_{n}}\right)^{\bullet t} \\
& =\left\{\left[\max \left(1-A_{n}^{\frac{t \varepsilon_{p}}{\eta}}, 0\right), \max \left(1-B_{n}^{\frac{t \varepsilon_{p}}{\eta}}, 0\right)\right],\right.
\end{aligned}
$$

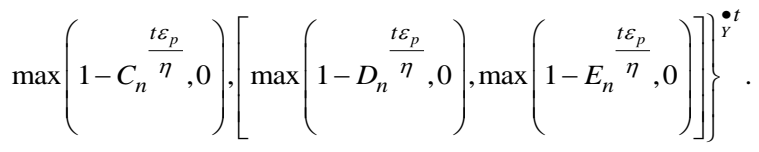

$$
\begin{aligned}
& =\left\{\left[\max \left(\left(1-A_{n}{ }^{\frac{t}{\eta}}\right)^{\varepsilon_{p}}, 0\right), \max \left(\left(1-B_{n}{ }^{\frac{t}{\eta}}\right)^{\varepsilon_{p}}, 0\right)\right], \max \left(\left(1-C_{n}{ }^{\frac{t}{\eta}}\right)^{\varepsilon_{p}}, 0\right),\right. \\
& \left.\left[\max \left(\left(1-D_{n}^{\frac{t}{\eta}}\right)^{\varepsilon_{p}}, 0\right), \max \left(\left(1-E_{n}^{\frac{t}{\eta}}\right)^{\varepsilon_{p}}, 0\right)\right]\right\} \text {. } \\
& =\left\{\left[\max \left(\left(1-A_{n}^{\frac{t \varepsilon_{p}}{\eta}}\right), 0\right), \max \left(\left(1-B_{n}^{\frac{t \varepsilon_{p}}{\eta}}\right), 0\right),\right.\right. \\
& \left.\max \left(\left(1-C_{n}^{\frac{t \varepsilon_{p}}{\eta}}\right), 0\right),\left[\max \left(\left(1-D_{n}^{\frac{t \varepsilon_{p}}{\eta}}\right), 0\right), \max \left(\left(1-E_{n}^{\frac{t \varepsilon_{p}}{\eta}}\right), 0\right)\right]\right\}
\end{aligned}
$$

Since $(16)=(17)$, hence the result.

\section{E. Theorem(Stability)}

$$
\begin{aligned}
& \text { If } t>0, \overline{M_{n+1}}=\left(\left[l_{M_{n+1}}, \overline{l_{M_{n+1}}}\right], c_{M_{n+1}},\left[\underline{r_{M_{n+1}}}, \overline{r_{M_{n+1}}}\right]\right) \text { then }
\end{aligned}
$$

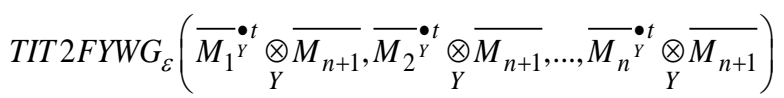

$$
=T I T 2 F Y W G_{\varepsilon}\left(\overline{M_{1}}, \overline{M_{2}}, \ldots, \overline{M_{n}}\right)_{Y}^{\bullet t} \underset{Y}{\otimes} \overline{M_{n+1}}
$$

Proof:

$$
\begin{aligned}
& \operatorname{TIT} 2 F Y W G_{\varepsilon}\left(\overline{M_{1}^{Y}}{ }_{Y}^{\bullet t} \otimes \overline{M_{n+1}}, \overline{M_{2}}{ }_{Y}^{\bullet t} \underset{Y}{\otimes} \overline{M_{n+1}}, \ldots, \overline{M_{n}^{Y}}{ }_{Y}^{\bullet t} \otimes \overline{M_{n+1}}\right) \\
& =\left\{\left[\max \left(1-A_{n}^{\frac{t \varepsilon_{p}}{\eta}}, 0\right) \underset{Y}{\otimes} \overline{M_{n+1}}, \max \left(1-B_{n}^{\frac{t \varepsilon_{p}}{\eta}}, 0\right) \underset{Y}{\otimes} \overline{M_{n+1}}\right],\right. \\
& \max \left(1-C_{n}^{\frac{t \varepsilon_{p}}{\eta}}, 0\right) \underset{Y}{\otimes} \overline{M_{n+1}}
\end{aligned}
$$

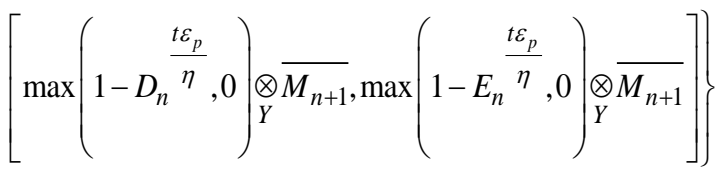$$
=\left\{\left[\max \left(1-\left[\underset{p=1}{n}\left(1-\max \left(1-\left[\operatorname{sum}_{q=\{p, n+1\}}\left(1-\underline{l_{M_{q}}}\right)^{\eta}\right]^{\frac{t}{\eta}}, 0\right)^{\varepsilon_{p}}\right)\right], 0\right),\right.\right.
$$$$
\left.\max \left(1-\left[\underset{p=1}{\operatorname{sum}}\left(1-\max \left(1-\left[\operatorname{sum}_{q=\{p, n+1\}}\left(1-\overline{l_{M_{q}}}\right)^{\eta}\right]^{\frac{t}{\eta}}, 0\right)^{\varepsilon_{p}}\right)\right], 0\right)\right]
$$$$
\max \left(1-\left[\underset{p=1}{n}\left(1-\max \left(1-\left[\operatorname{sum}_{q=\{p, n+1\}}\left(1-c_{M_{q}}\right)^{\eta}\right]^{\frac{t}{\eta}}, 0\right)^{\varepsilon_{p}}\right)\right], 0\right) \text {, }
$$$$
\left[\max \left(1-\left[\underset{p=1}{n}\left(1-\max \left(1-\left[\operatorname{sum}_{q=\{p, n+1\}}\left(1-\underline{r_{M_{q}}}\right)^{\eta}\right]^{\frac{t}{\eta}}, 0\right)^{\varepsilon_{p}}\right]\right), 0\right),\right.
$$$$
\left.\left.\max \left(1-\left[\underset{p=1}{n}\left(1-\max \left(1-\left[\operatorname{sum}_{q=\{p, n+1\}}\left(1-\overline{r_{M_{q}}}\right)^{\eta}\right]^{\frac{t}{\eta}}, 0\right)^{\varepsilon_{p}}\right]\right), 0\right)\right]\right\} \text {. }
$$$$
=\left\{\left[\max \left(\left[1-A_{n}^{\frac{\varepsilon_{p}}{\eta}}\right)+\left(\left[\left(1-\underline{l_{M+1}}\right)^{\eta}\right]^{\frac{1}{\eta}}\right)^{\substack{n \\ \text { sum } \\ p=1}}, 0\right),\right.\right.
$$ 


$$
\begin{aligned}
& \left.\max \left(\left(1-B_{n}^{\frac{\varepsilon_{p}}{\eta}}\right)+\left(\left[\left(1-\overline{l_{M+1}}\right)^{\eta}\right]^{\frac{1}{\eta}}\right)^{\substack{n \\
p=1}}, 0\right)\right] \text {, } \\
& \max \left(\left(1-C_{n} \frac{\varepsilon_{p}}{\eta}\right)+\left(\left[\left(1-c_{M+1}\right)^{\eta}\right]^{\frac{1}{\eta}}\right)^{\substack{n \\
\text { sum }}} \varepsilon_{p}, 0\right), \\
& \max \left(\left(1-D_{n}^{\frac{\varepsilon_{p}}{\eta}}\right)+\left(\left[\left(1-\underline{r_{M+1}}\right)^{\eta}\right]^{\frac{1}{\eta}}\right)^{\substack{n \\
\text { sum }}}, 0\right), \\
& \left.\left.\max \left(\left(1-E_{n} \frac{\varepsilon_{p}}{\eta}\right)+\left(\left[\left(1-\overline{r_{M+1}}\right)^{\eta}\right]^{\frac{1}{\eta}}\right)^{\substack{n u m \\
p=1}}, 0\right)\right]\right\} \text {. }
\end{aligned}
$$

Based on the theorem A and the operational law,

$\operatorname{TIT}^{2} \mathrm{FYWG}_{\varepsilon}\left\langle\overline{M_{1}}, \overline{M_{2}}, \ldots, \overline{M_{n}}\right\rangle^{\stackrel{\bullet}{Y}} \underset{Y}{\otimes} \overline{M_{n+1}}$

$$
\begin{aligned}
& \left\{\left[\max \left(1-A_{n}^{\frac{t \varepsilon_{p}}{\eta}}, 0\right), \max \left(1-B_{n}^{\left.\left.\frac{t \varepsilon_{p}}{\eta}, 0\right)\right],}\right.\right.\right. \\
& \left.\max \left(1-C_{n}^{\frac{t \varepsilon_{p}}{\eta}}, 0\right),\left[\max \left(1-D_{n}^{\frac{t \varepsilon_{p}}{\eta}}, 0\right), \max \left(1-E_{n}^{\left.\frac{t \varepsilon_{p}}{\eta}, 0\right)}\right)\right]\right\} \\
& \underset{Y}{\otimes}\left\langle\left[l_{M_{n+1}}, \overline{l_{M_{n+1}}}\right], c_{M_{n+1}},\left[\underline{r_{M_{n+1}}}, \overline{r_{M_{n+1}}}\right]\right\rangle
\end{aligned}
$$$$
=\left\{\left[\max \left(\left(1-A_{n}^{\frac{\varepsilon_{p}}{\eta}}\right)+\left(\left[\left(1-\underline{l_{M+1}}\right)^{\eta}\right]^{\frac{1}{\eta}}\right), 0\right),\right.\right.
$$$$
\left.\max \left(\left(1-B_{n} \frac{\varepsilon_{p}}{\eta}\right)+\left(\left[\left(1-\overline{l_{M+1}}\right)^{\eta}\right]^{\frac{1}{\eta}}\right), 0\right)\right],
$$$$
\max \left(\left(1-C_{n}^{\frac{\varepsilon_{p}}{\eta}}\right)+\left(\left[\left(1-c_{M+1}\right)^{\eta}\right]^{\frac{1}{\eta}}\right), 0\right) \text {, }
$$$$
\left[\max \left(\left(1-D_{n}^{\frac{\varepsilon_{p}}{\eta}}\right)+\left(\left[\left(1-\underline{r}_{M+1}\right)^{\eta}\right]^{\frac{1}{\eta}}\right), 0\right),\right.
$$

$$
\left.\left.\max \left(\left(1-E_{n} \frac{\varepsilon_{p}}{\eta}\right)+\left(\left[\left(1-\overline{r_{M+1}}\right)^{\eta}\right]^{\frac{1}{\eta}}\right), 0\right]\right)\right\}
$$

Here, $(19)=(20)$.

Hence the result.

\section{F. Theorem(Image Contrast)}

For given arguments $\bar{M}_{p}, p=1,2, \ldots, n$ and the parameter $\eta \in(1,+\infty)$ then TIT2FYWG operator is monotonically nondecreasing (MND) with respect to the parameter.

Proof:

To prove the operator is MND with respect to the parameter, we have to prove the same for every reference point function is MND w.r.t the parameter.

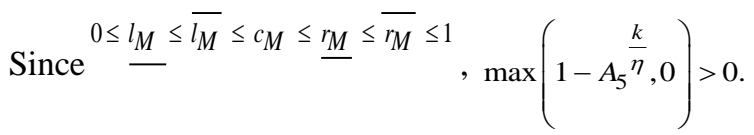

And it is true for all the reference points.

Hence the result.

Note: The above theorems also can be proved by using TIT2FYWA operator.

\section{THEORY OF IMAGE PROCESSING AND ROLE OF YAGER NORMS}

The advantage of considering Yager triangular norms is having maximum and minimum operators which will be much useful in Image Processing while filtering.

\section{A. Associativity of Yager T Norms [8]}

Each fuzzy norm should be satisfied the associativity property to compute the norm for more than two values using continual manner as follows.

Consider the associativity property for Yager $\mathrm{T}$ Norm (YTN)

$$
\begin{aligned}
& Y T N\left[\operatorname{TN}\left(\bar{M}_{1}, \bar{M}_{2}\right), \bar{M}_{3}\right] \\
& =\max \left\{1-\left[\left[1-T\left(\bar{M}_{1}, \bar{M}_{2}\right)\right]^{\eta},\left(1-\bar{M}_{3}\right)^{\eta}\right]^{1 / \eta}, 0\right\} \\
& =\max \left\{1-\left\{1-\left[\max \left\{1-\left[\left[\left(1-\bar{M}_{1}\right)^{\eta}+\left(1-\bar{M}_{2}\right)^{\eta}\right]^{1 / \eta}\right]^{\eta}, 0\right\}\right]+\left(1-\bar{M}_{3}\right)^{\eta}\right\}^{1 / \eta}, 0\right\} \\
& \left.=\max \left\{\left\{1-1+\left\{\max \left[1-\left(\left(1-\bar{M}_{1}\right)^{\eta}+\left(1-\bar{M}_{2}\right)^{\eta}\right)^{1 / \eta}\right]^{\eta}, 0\right\}+\left(1-\bar{M}_{3}\right)^{\eta}\right\}^{1 / \eta}\right], 0\right\}
\end{aligned}
$$




$$
\begin{aligned}
& \left.=\max \left\{\left\{\max \left\{\left[1-\left(\left(1-\bar{M}_{1}\right)^{\eta}+\left(1-\bar{M}_{2}\right)^{\eta}\right)^{1 / \eta}\right]^{\eta}, 0\right\}+\left(1-\bar{M}_{3}\right)^{\eta}\right\}\right], 0\right\} \\
& \left.=\max \left\{\left[1-\left(\left(1-\bar{M}_{1}\right)^{\eta}+\left(1-\bar{M}_{2}\right)^{\eta}\right)^{1 / \eta}\right]^{\eta}+\left(1-\bar{M}_{3}\right)^{\eta}\right]^{1 / \eta}, 0\right\} \\
& =\max \left\{\left(\left[1-\left(1-\bar{M}_{1}\right)^{\eta}+\left(\left(1-\bar{M}_{2}\right)^{\eta}+\left(1-\bar{M}_{3}\right)^{\gamma}\right)^{1 / \eta}\right]^{\eta}\right]^{1 / \eta}, 0\right\} \\
& =\operatorname{YTN}\left[\bar{M}_{1}, T N\left(\bar{M}_{2}, \bar{M}_{3}\right)\right] .
\end{aligned}
$$

Similarly for Yager T conorm (YTCN).

Here we can consider $\bar{M}_{1}$ and $\bar{M}_{2}$ as the Interval Type-2 Triangular Fuzzy Number. If $\max \left(\bar{M}_{1}, \bar{M}_{2}\right)=1$ then (YTN) will become $\min \left(\bar{M}_{1}, \bar{M}_{2}\right)$. If $\min \left(\bar{M}_{1}, \bar{M}_{2}\right)=0$ then (YTCN) will become $\max \left(\bar{M}_{1}, \bar{M}_{2}\right)$. In the same manner we can have for YTCN. Here the definition of YTN accords the inference around the effectiveness of the norm and $\gamma$, its complimentary parameter. Generally, it allows tuning between the norms.

If $\eta$ approaches 0 , then YTN will be $\min \left(\bar{M}_{1}, \bar{M}_{2}\right)$ only when $\max \left(\bar{M}_{1}, \bar{M}_{2}\right)=1$ i.e., their drastic product. If $\eta$ approaches 1 , then YTN becomes $\max \left[\left(\bar{M}_{1}+\bar{M}_{2}-1\right), 0\right]$.

B. Role of Associativity of Yager T Norm in Image Processing

Using Fuzzy Set approach, we can generalize a binary morphology into MFM. Morphological operations are the basic tools to modify the image $I_{1}$ over the structural aspect of $I_{2}$. To study the structure of $I_{1}$, size and shape of the $I_{2}$ are chosen accordingly.

\section{Morphological Operations [24]}

(i). Erosion (Maximum)

(ii). Dilation (Minimum)

(iii). Opening and Closing (Idempotency)

\section{Role of T-Norms in Image Processing [17]}

For constructing FM, we use Conjunctions and Implications. Among these two, we used conjunctions (tnorms) here and from the below, the representation of mathematical properties in image processing has been explained.

\section{1) Commutativity:}

The result of IDS application on two successive points $\mathrm{P}$ and $\mathrm{Q}$ is the same as applying on them in inverse order, since the value of flapped points is the sum of values of all data diluted on that point and therefore the operator is commutative.

\section{2) Monotonicity:}

If the brightness of $\mathrm{P}$ is less than or equal to $\mathrm{Q}$ then all the data points in brightness of $\mathrm{P}$ is less than or equal to brightness with respect to the corresponding data points of brightness of Q.

Therefore for any point ${ }_{n}$, the brightness appeared from $\mathrm{P}$ is $n+a P$, where $a$ is proportional to inverse of distance. Similarly, the brightness appears from $\mathrm{Q}$ is $n+a Q$ Since $a>0$, the brightness of $n$ appeared from $\mathrm{P}$ is less than or equal to that of from $\mathrm{Q}$.

\section{3) Associativity:}

Assume that $\mathrm{P}, \mathrm{Q}$ and $\mathrm{R}$ are the sources of light going to affect to the point $n$ by IDS.

For every source, IDS increases the brightness with respect to the distance regardless of other sources.

On the point $n$, the order of applying IDS does not affect the distance.

Sum of effects of $\mathrm{P}, \mathrm{Q}$ and $\mathrm{R}$ is the value of $n$. Therefore, the operator is associative.

\section{4) Idempotency:}

This property and its generalization is used for the morphological operation opening and closing.

\section{5) Neutrality of 0 :}

Consider a pyramid of height 0 , sum of this with others does not influence them. Therefore, 0 is the neutral element.

\section{E. Morphological Gradient (MG) [24]}

It is useful to detect an edge and act as a first approximation to a morphological segmentation. MG is the discrepancy between

\section{a) dilation and erosion \\ b) dilation and the original image \\ c) original image and its erosion}

\section{F. T-Norm and Image Compression(IC) [17]}

IC is based on Fuzzy Relational (FR) Equations and it is a grayscale image of size $C \times D$ as a FR $\mathfrak{R} \in \mathrm{F}(A, B)$ where, $A=\left\{a_{1}, a_{2}, \ldots, a_{C}\right\}, B=\left\{b_{1}, \mathrm{~b}_{2}, \ldots, \mathrm{b}_{D}\right\}$ enclosed the depth range of each pixel into $[0,1]$.

$$
C S=\left\{C S^{(R)}, C S(G), C S(B)\right\} \subset \mathrm{F}(A, B) \text { represent the color }
$$
image on RGB Color Space (CS). Here $C S^{(R)}, C S^{(G)}$ and $C S^{(B)}$ are the Red, Green and Blue color spaces.

For clarity, gray scale image (GSI) will be considered. The GSI $\Re \in \mathrm{F}(A, B)$ is restrict into $\wp \in \mathrm{F}(I \times J)$, through a max t- 
norm FR equations with composition $\wp=\max _{b \in B}\left\{V_{j}(b) T N \max _{a \in A}\left[U_{i}(a) T N \square(a, b)\right]\right\}$, where $\mathrm{TN}$ is a continuous t-norm, $\quad U_{i} \in U=\left\{U_{1}, U_{2}, \ldots, U_{I}\right\} \subset \mathrm{F}(A) \quad$ and $V_{i} \in V=\left\{V_{1}, \mathrm{~V}_{2}, \ldots, \mathrm{V}_{J}\right\} \subset \mathrm{F}(B)$ are the coders.

The shape of the FSs of coders are the triangular line, preferable for IC. We can adjust the compression rate of IC by the sum of FSs consist in $U$ and $V$ ad is defined by $\zeta=\frac{I J}{A B}$. Here $I J$ and $A B$ the compressed and original image coefficients respectively. By adjusting the parameter $\eta$, YTN will all the continuous T-norm where as Zadeh's and major t norms cannot do the same. Though Frank t-norm can do the same, due to the computational complexity, we prefer Yager's t norm for image processing.

\section{G. Role of T2FS [24]}

Here the components of an image processing and the role of T2FS is correlated.

\section{1) Image Contrast Enrichment:}

The most common image enrichment method is histogram equalization. Since an image has an imprecise pixel grey values, it may not produce acceptable results in IP. To handle the ambiguity of the gray values, Fuzzy methods have been suggested by many researchers.

By adjusting the membership values, the contrast of the image is increased by contrast intensification operator and it transforms the higher MVs to much higher and lower MVs to much lower in a nonlinear aspect. Since this aspect considers whole image, global histogram fails to produce satisfactory results.

Though the fuzzy methods deals ambiguity well and produced proper enrichment, it fails in some case and hence T2FS has been considered for this purpose since it deals more uncertainties.

\section{2) Image Segmentation:}

Region boundaries of an image may not have a fine growth, therefore fuzzy decision is used to check whether the pixel exists to a region and T2FS may be applied to get better threshold images.

\section{3) Clustering:}

The images have different regions with different pitch, clustering collects the similar pixels in a group with membership value 1 and collects different pixels in different group with membership value 0 . But in fuzzy clustering the pixel associate to different number of groups and hence the MVs are not 0 .

\section{4) Edge Detection:}

Since most of the images have poor brightness, the proper decision cannot be taken in checking the existence of an edge in an image. Edges may be enriched before carrying out the edge detection. In taking off the edge due to ambiguity, fuzzy method may be useful and may not find better edges. At this junction T2FS is useful as it handles more uncertainties.

\section{5) Morphology:}

Which is a non-linear image processing technique and is used to shape the image features. Here also T2FS plays an important role to get better results.

\section{APPLICATION OF IMAGE PROESSING}

Fig. 2. Shows that the Architecture has been proposed for edge detection on DICOM image using triangular norms

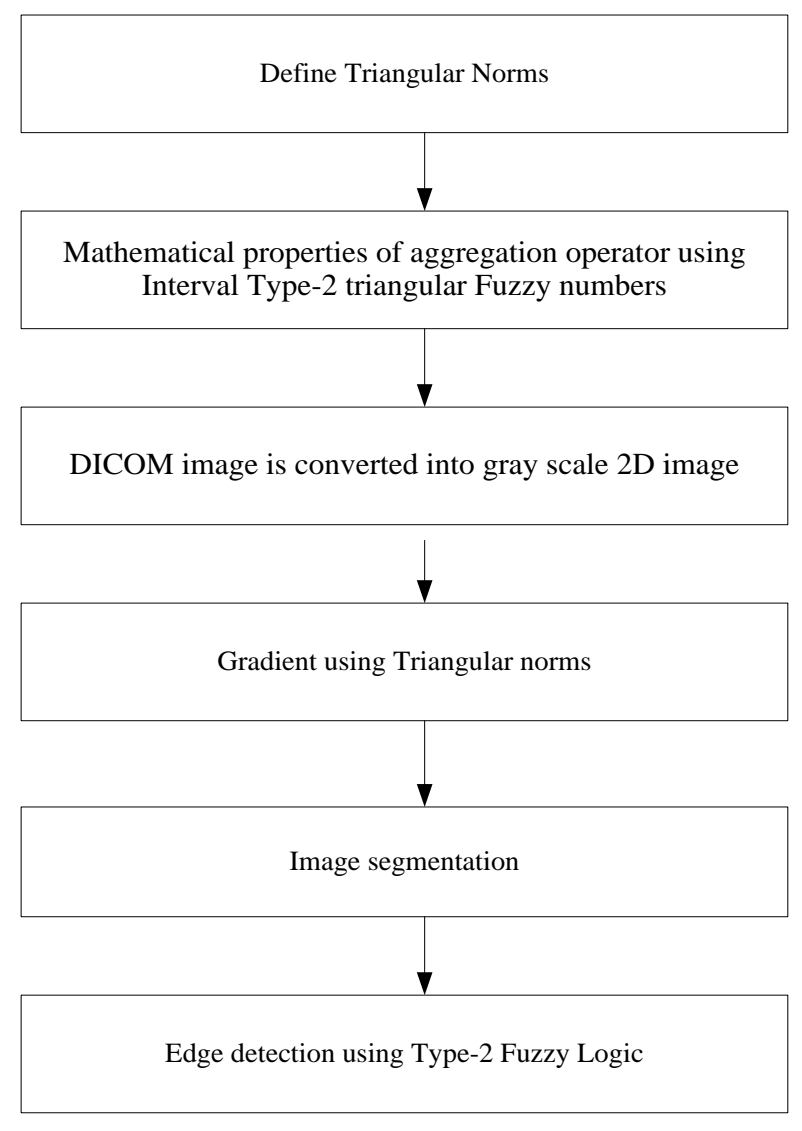

Fig. 2. Architecture for Edge Detection.

Using MATLAB 2015a, triangular norms has been applied in medical image processing from a patient DICOM image. In this case $3 \mathrm{D}$ image is converted to $2 \mathrm{D}$ image.

In Fig. 3, the image is collected from our experimental data set from a patient DICOM image in the Fig. 7. From this Fig. 7. the clear image Fig. 8. Has been considered for the experiment.

$$
\begin{array}{ll}
\text { Size of the image } & =512 \times 517 . \\
\text { Mean of the image } & =28.83 . \\
\text { Standard deviation } & =60.79
\end{array}
$$

Mean absolute deviation $=40.03$.

To identify the gradient of the image by dilation-erosion, triangular norms are used. 


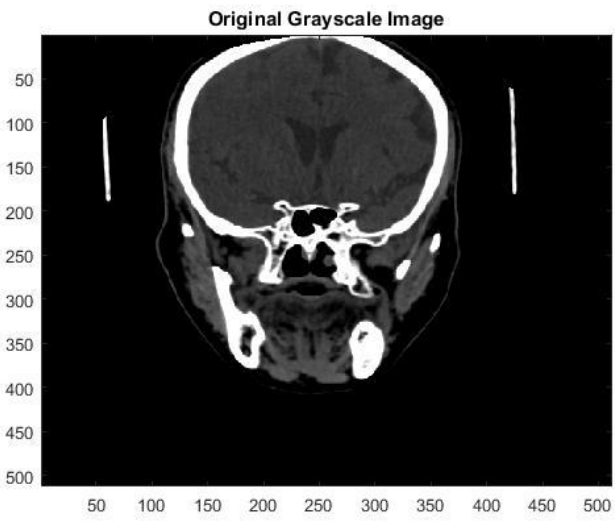

Fig. 3. Original gray scale DICOM image.

Structuring elements are used in gradient value.

Image Erosion is
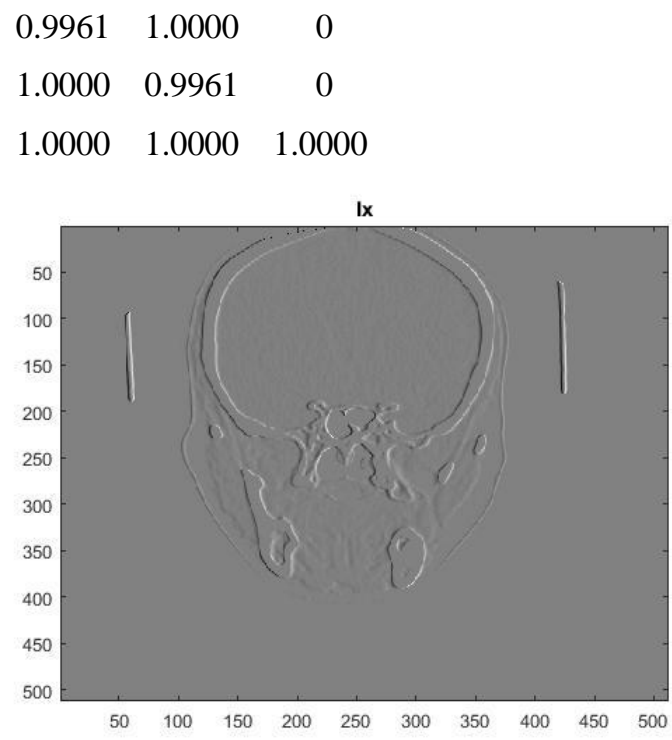

Fig. 4. Gradient through $\mathrm{x}$ axis.

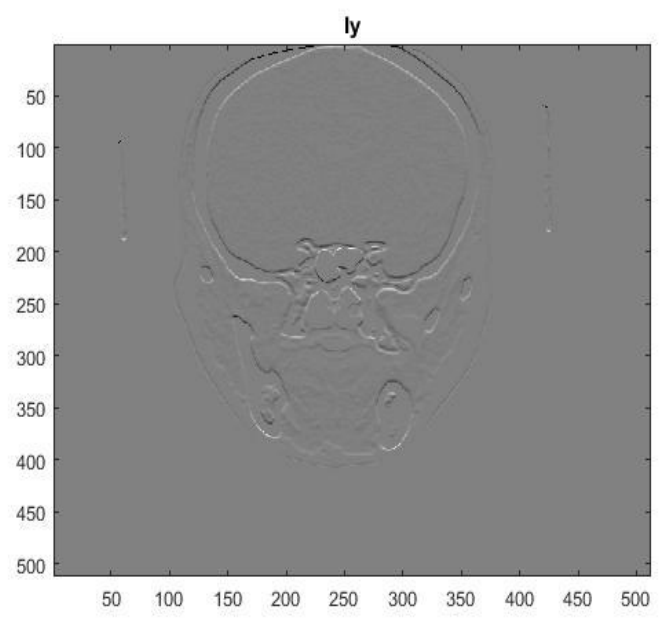

Fig. 5. Gradient through y axis.
Gradient through y axis. The below figures are the output of the image processing application in edge detection through triangular norms by MATLAB $2015 \mathrm{a}$.

Fig. 4. is the gradient through $\mathrm{x}$ axis and Fig. 5. is the gradient through the y axis.

The figures reveals that the image gradient to identify the region uniformly.

Fig. 6. is the output of the edge detection through T2 fuzzy by our experimental output using MATLAB 2015a

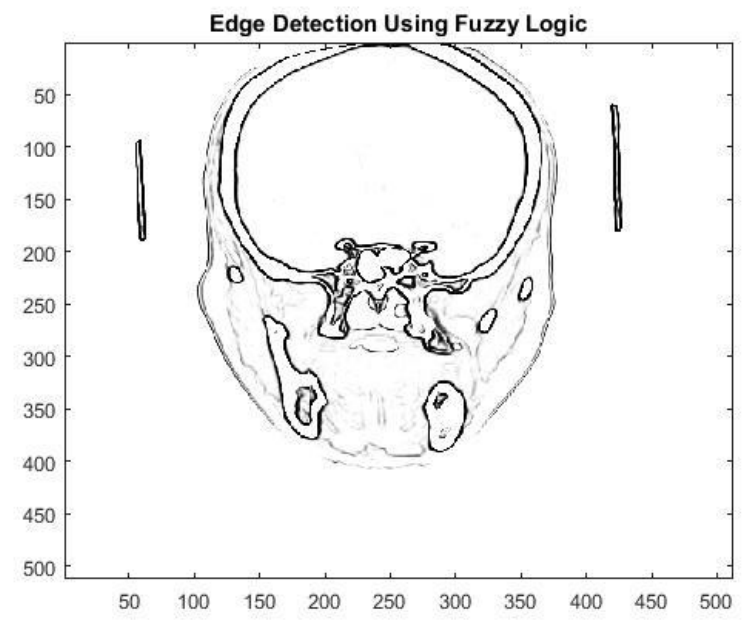

Fig. 6. Edge detection image.

Fig. 6. shows that the edges of the object through FIS and equating the pixel on both direction. If the edge is block then pixel is not 0 .

Edge detection plays a vital role in image identification. It is observed that, fuzzy logic edge detector helps in reducing the memory for saving medical images.

\section{CONCLUSION}

In this paper, operational laws of addition, multiplication, power and multiplication by an arbitrary number using Yager triangular norms for TIT2FN are derived. Also some properties of aggregation operation using Fuzzy Yager Weighted Geometric operators have been proved. Since Yager aggregation operator contains minimum and maximum operator, it will be act as a morphological filters in medical image processing. Detailed representation of the mathematical properties in image processing is presented. Also, the gradient of the DICOM image of MRI scan of a patient using Triangular norms is found and done edge detection using MATLAB in T2 fuzzy logic. The future work is planned to apply T2 Fuzzy logic in edge extraction on medical image in 3D models.

\section{Data Availability statement}

The DICOM data used to support the findings of this study are available from the corresponding author upon request.

\section{Conflict of interest}

The authors declare that they have no conflict of interest. 


\section{Supplementary Materials}

The data set in Fig. 7. is the montage of the images in a single file and is from a patient MRI. This MRI which is in the $3 \mathrm{D}$ form is converted to $2 \mathrm{D}$ form (DICOM) using MATLAB2015a. The 3D format consists of 25 DICOM file formats; the montage of the images is obtained as a single frame. Out of these 25 DICOM images a clear full image is chosen as in Fig. 8. Using Dilation corrosion method, the gradient is identified. The edge detection is performed through triangular norms using MATLAB 2015a.

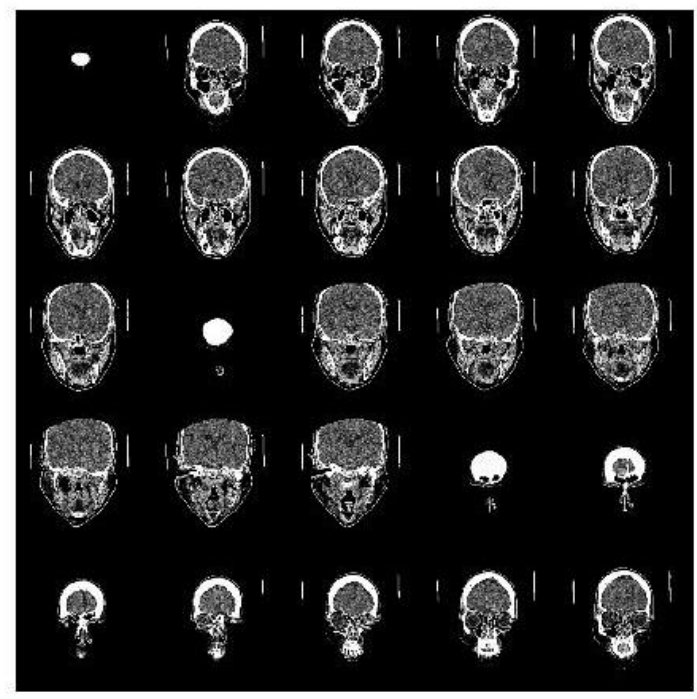

Fig. 7. Montage of the images.

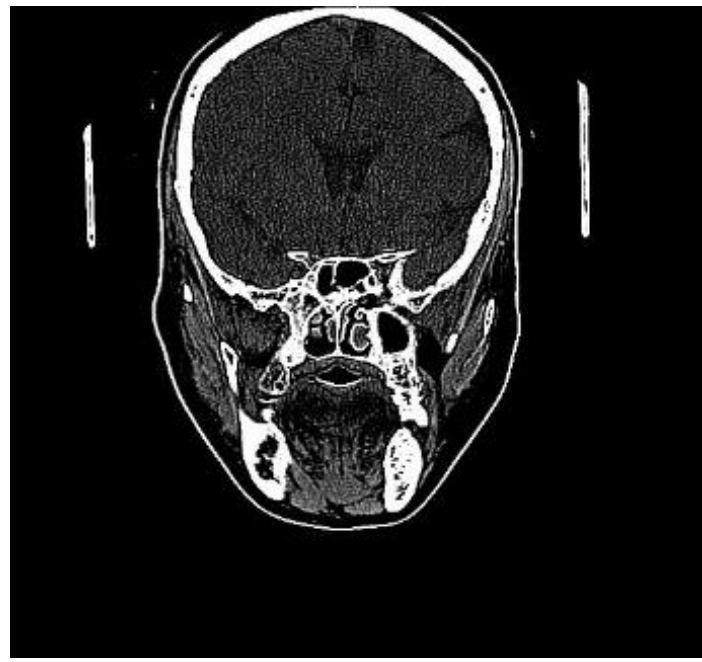

Fig. 8. Clear image from montage.

\section{REFERENCES}

[1] T. Calvo, G. Mayor and R. Messiar, "Aggregation Operators: New Trends and Application," 2012, Springer Publications.

[2] D. Vivona and M. Divari, "On Information Reception and Some of its Aggregation Operators," 8th International Summer School on Aggregation Operators, Kotowice, Poland, University of Silesia, 2015, pp.233-237.

[3] J. Qin and X. Liu, "Frank Aggregation operators for Triangular Interval Type-2 Fuzzy Set and its Application in Multiple Attribute Group
Decision Making," Journal of Applied Mathematics, Vol. 1, 2014, pp.124.

[4] F. Castro-Company and P. Tirado, "On Yager and Hamacher t-norms and Fuzzy Metric spaces," International Journal of Intelligent Systems, Vol.29, No.12, 2014, pp.1173-1180.

[5] E. Czogala and H. Zimmermann, "The Aggregation Operators for Decision Making in Probabilistic Fuzzy Environment," Fuzzy Sets and Systems, Vol.9, No.1, 1984, pp.183-196.

[6] T. Calvo and R. Mesiar, "Weighted Means Based on Triangular Conorms," International Journal of Uncertain Fuzziness Knowledge Based Systems, Vol.29, No.12, 2001, pp.1173-1180.

[7] M. M. Gupta and J. Qi, "Theory of t-norms and Fuzzy Inference Methods," Fuzzy Sets and Systems, Vol. 40, No. 3, 1991, pp.431-450.

[8] D. Dubois and H. Prade, "On the Use of Aggregation operations in Information Fusion Processes," Fuzzy Sets and Systems, Vol. 142, No.3, 2004, pp.143-161.

[9] M. Detynieki, "Mathematical Aggregation Operators and their Application to Video Querying," Ph.D. Thesis in Artificial Intelligence Specialty of Paris, 2000, pp.1-104.

[10] R. Mesiar, A. Kolesarova, T. Calvo and M. Komornikova, "A review of aggregation functions," In: Fuzzy Sets and Their Extensions: Representation, Aggregation and Models, H.Bustince et al. (Eds.), Springer, Berlin, 2008, pp.121-144.

[11] D. Dubois and H. Prade, "A review of fuzzy set aggregation Connectives, Information Sciences," Vol. 36, No.1-2, 1985, pp. 85-121.

[12] R. R. Yager, "Aggregation operators and fuzzy systems modeling," Fuzzy Sets and Systems, Vol. 67, No.2, 1994, pp.129-145.

[13] L. A. Zadeh, "The concept of a linguistic variable and its application to Approximate Reasoning-I," Information Sciences, Vol.8, No.3, 1975, pp.199-249.

[14] C. H. Chiu and W. J. Wang, "A simple computation of MIN and MAX operations for fuzzy numbers," Fuzzy Sets and Systems, Vol.126, No. 2, 2002, pp.273-276.

[15] Z. Gera and J. Dombi, "Exact calculations of extended logical operations on fuzzy truth values," Fuzzy Sets and Systems, Vol.159, No.11, 2008, pp.1309-1326.

[16] S. Zhou, F. Chiclana, R. John and J. Garibaldi, "Type-1 OWA operators for aggregating uncertain information with uncertain weights induced by type-2 linguistic Quantities," Fuzzy Sets and Systems, Vol.159, No.24, 2008, pp.3281-3296.

[17] K Franke, M. Koppen and B.Nickolay, "Fuzzy Image Processing by using Dubois and Prade Fuzzy Norms," Pattern Recognition, 2000, pp.14.

[18] A. A. Kiaei, S. B. Shouraki, S.H. Khasteh, M. Khademi and A. R. G. Samani, "New S-norm and T-norm Operators for Active Learning Method," Fuzzy Optimization and Decision Making, 2010, pp.1-11.

[19] J. Dombi, "A general class of fuzzy operators, the DeMorgan class of fuzzy operators and fuzziness measures induced by fuzzy operators," Fuzzy Sets and Systems, Vol. 8, No.2, 1982, pp.149-163.

[20] Y. Yao and J. Wang, "Interval based Uncertain Reasoning using Fuzzy and Rough Sets," Advances in Machine Intelligence \& Soft-Computing, 1996, pp.1-20.

[21] K. Franke and M. Koppen, "A computer-based system to support forensic studies on handwritten documents," International Journal on Document Analysis and Recognition, 2000, pp.1-13.

[22] K. Hirota, H. Nobuhara, K. Kawamoto and S. I. Yoshida, "On a Lossy Image Compression/ Reconstruction Method Based on Fuzzy Relational Equations," Iranian Journal of Fuzzy Systems, Vol.1, No.1, 2004, pp.3342.

[23] M. G. Hidalgo, A. M. Torres, D. R. Aguilera, J. T. Sastre, "Image Analysis Applications of Morphological Operators on Uninorms," Soft computing techniques for uncertainty management in image processing, 2009, pp. 630-635. ISBN: 978-989- 95079-6-8.

[24] T. Chaira, "Medical Image Processing: Advanced Fuzzy Set Theoretic Techniques," CRC Publications, 2015, ISBN: 9781498700450CAT\#K24522. 
[25] O. Castillo, M. A. Sanchez, C. I. Gonzalez and G. E. Martinez, "Review of Recent Type-2 Fuzzy Image Processing Applications," Information, Vol. 8, No. 97, 2017, pp. 1-18.

[26] I. Sheikh and K. A. Khan, "Simulation of Image Edge Detection using Fuzzy Logic in MATLAB," International Journal of Computer \& Mathematical Sciences, Vol. 6, No. 5, 2017, pp. 19-22.

[27] Kenjharayoobchandio and Yasarayaz, "Fuzzy Logic Based Digital Image Edge Detection," International Journal of Electrical, Electronics and Data Communication, Vol. 6, No. 2, 2018, pp.18-22.
[28] A. K. Dutta, "Intuitionistic Fuzzy Logic Implementation in Image Fusion Technique," Asian Journal of Research in Computer Science, Vol. 1. No. 1, 2018, pp. 1-7.

[29] D. G. Privezentsev, A. L. Zhiznyakov, A. V. Astafiev and E. V. Pugin, "Using fuzzy fractal features of digital images for the material surface analysis," Journal of Physics, Vol. 944, 2018, pp. 1-5.

[30] R. Maini and H. Aggarwal, "Study and Comparison of Various Image Edge Detection Techniques," International Journal of Image Processing, Vol.3, No.1, 2009, pp.1-12. 